\title{
Load-balancing scatter operations for grid computing
}

\author{
Stéphane Genaud ${ }^{\mathrm{a}}$, Arnaud Giersch ${ }^{\mathrm{a}, *}$, Frédéric Vivien ${ }^{\mathrm{b}}$ \\ ${ }^{a}$ ICPS/LSIIT, UMR CNRS-ULP 7005, Parc d'Innovation, Bd Sébastien Brant, \\ BP 10413, 67412 Illkirch Cedex, France \\ b LIP, UMR CNRS-ENS Lyon-INRIA-UCBL 5668, École normale supérieure de Lyon, \\ 46 allée d'Italie, 69364 Lyon Cedex 07, France
}

Received 18 March 2003; revised 28 May 2004; accepted 16 July 2004

\begin{abstract}
We present solutions to statically load-balance scatter operations in parallel codes run on grids. Our load-balancing strategy is based on the modification of the data distributions used in scatter operations. We study the replacement of scatter operations with parameterized scatters, allowing custom distributions of data. The paper presents: (1) a general algorithm which finds an optimal distribution of data across processors; (2) a quicker guaranteed heuristic relying on hypotheses on communications and computations; (3) a policy on the ordering of the processors. Experimental results with an MPI scientific code illustrate the benefits obtained from our load-balancing.

(c) 2004 Elsevier B.V. All rights reserved.

Keywords: Parallel programming; Grid computing; Heterogeneous computing; Load-balancing; Scatter operation
\end{abstract}

\footnotetext{
${ }^{*}$ Corresponding author. Tel.: +33 3902445 42; fax: +33 390244547.

E-mail addresses: stephane.genaud@icps.u-strasbg.fr (S. Genaud), arnaud.giersch@icps.u-strasbg.fr (A. Giersch), frederic.vivien@ens-lyon.fr (F. Vivien).
} 


\section{Introduction}

Traditionally, users have developed scientific applications with a parallel computer in mind, assuming an homogeneous set of processors linked with an homogeneous and fast network. However, grids [16] of computational resources usually include heterogeneous processors, and heterogeneous network links that are orders of magnitude slower than in a parallel computer. Therefore, the execution on grids of applications designed for parallel computers usually leads to poor performance as the distribution of workload does not take the heterogeneity into account. Hence the need for tools able to analyze and transform existing parallel applications to improve their performances on heterogeneous environments by load-balancing their execution. Furthermore, we are not willing to fully rewrite the original applications but we are rather seeking transformations which modify the original source code as little as possible.

Among the usual operations found in parallel codes is the scatter operation, which is one of the collective operations usually shipped with message passing libraries. For instance, the mostly used message passing library MPI [26] provides a MPI_Scatter primitive that allows the programmer to distribute even parts of data to the processors in the MPI communicator.

The less intrusive modification enabling a performance gain in an heterogeneous environment consists in using a communication library adapted to heterogeneity. Thus, much work has been devoted to that purpose: for MPI, numerous projects including MagPIe [23], MPI-StarT [20], and MPICH-G2 [22], aim at improving communications performance in presence of heterogeneous networks. Most of the gain is obtained by reworking the design of collective communication primitives. For instance, MPICH-G2 performs often better than MPICH to disseminate information held by a processor to several others. While MPICH always use a binomial tree to propagate data, MPICH-G2 is able to switch to a flat tree broadcast when network latency is high [21]. Making the communication library aware of the precise network topology is not easy: MPICH-G2 queries the underlying Globus [15] environment to retrieve information about the network topology that the user may have specified through environment variables. Such network-aware libraries bring interesting results as compared to standard communication libraries. However, these improvements are often not sufficient to attain performance considered acceptable by users when the processors are also heterogeneous. Balancing the computation tasks over processors is also needed to really take benefit from grids.

The typical usage of the scatter operation is to spawn an SPMD computation section on the processors after they received their piece of data. Thereby, if the computation load on processors depends on the data received, the scatter operation may be used as a means to load-balance computations, provided the items in the data set to scatter are independent. MPI provides the primitive MPI_Scatterv that allows to distribute unequal shares of data. We claim that replacing MPI_Scatter by MPI_Scatterv calls parameterized with clever distributions may lead to great performance improvements at low cost. In terms of source code rewriting, the transformation of such operations does not require a deep source code re-organization, and 
it can easily be automated in a software tool. Our problem is thus to load-balance the execution by computing a data distribution depending on the processors speeds and network links bandwidths.

In Section 2 we present our target application, a real scientific application in geophysics, written in MPI, that we ran to ray-trace the full set of seismic events of year 1999. In Section 3 we present our load-balancing techniques, in Section 4 the processor ordering policy we derive from a case study, in Section 5 our experimental results, in Section 6 the related works, and we conclude in Section 7.

\section{Motivating example}

\subsection{Seismic tomography}

The geophysical code we consider is in the seismic tomography field. The general objective of such applications is to build a global seismic velocity model of the Earth interior. The various velocities found at the different points discretized by the model (generally a mesh) reflect the physical rock properties in those locations. The seismic waves velocities are computed from the seismograms recorded by captors located all around the globe: once analyzed, the wave type, the earthquake hypocenter, and the captor locations, as well as the wave travel time, are determined.

From these data, a tomography application reconstructs the event using an initial velocity model. The wave propagation from the source hypocenter to a given captor defines a path, that the application evaluates given properties of the initial velocity model. The time for the wave to propagate along this evaluated path is then compared to the actual travel time and, in a final step, a new velocity model that minimizes those differences is computed. This process is more accurate if the new model better fits numerous such paths in many locations inside the Earth, and is therefore very computationally demanding.

\subsection{The example application}

We now outline how the application under study exploits the potential parallelism of the computations, and how the tasks are distributed across processors. Recall that the input data is a set of seismic waves characteristics each described by a pair of 3D coordinates (the coordinates of the earthquake source and those of the receiving captor) plus the wave type. With these characteristics, a seismic wave can be modeled by a set of ray paths that represents the wavefront propagation. Seismic wave characteristics are sufficient to perform the ray-tracing of the whole associated ray path. Therefore, all ray paths can be traced independently. The existing parallelization of the application (presented in [19]) assumes an homogeneous set of processors (the implicit target being a parallel computer). There is one MPI process per processor. The following pseudo-code outlines the main communication and computation phases: 


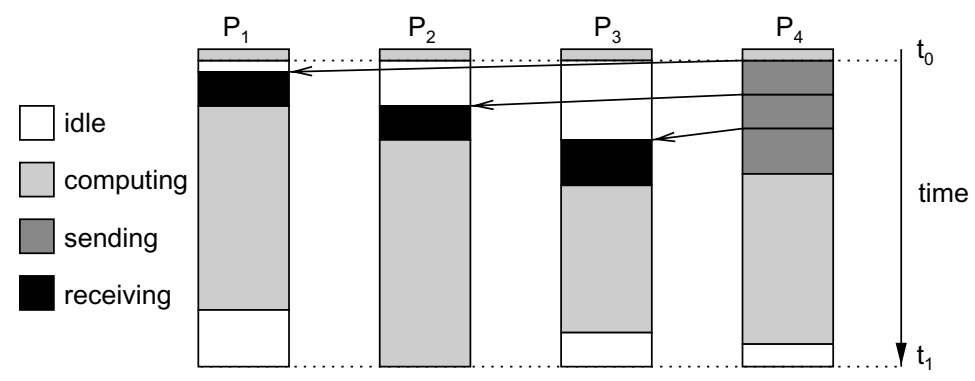

Fig. 1. A scatter communication followed by a computation phase.

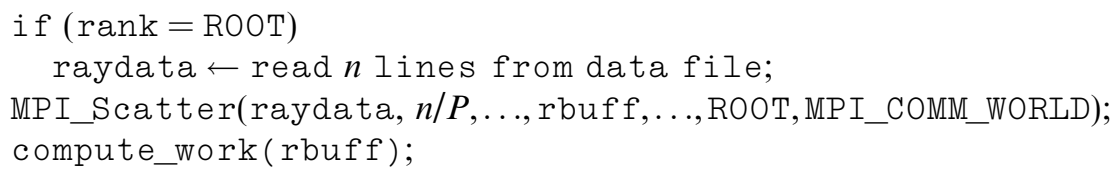

where $P$ is the number of processors involved, and $n$ the number of data items. The MPI_Scatter instruction is executed by the root and the computation processors. The processor identified as ROOT performs a send of contiguous blocks of $\lfloor n / P\rfloor$ elements from the raydata buffer to all processors of the group while all processors make a receive operation of their respective data in the rbuff buffer. For sake of simplicity the remaining $(n \bmod P$ ) items distribution is not shown here. Fig. 1 shows a potential execution of this communication operation, with $P_{4}$ as root processor.

\subsection{Communication model}

Fig. 1 outlines the behavior of the scatter operation as it was observed during the application runs on our test grid (described in Section 5.1). In the MPICH-G2 implementation we used (v1.2.2.3), when it performs a scatter operation, the root process $\left(P_{4}\right.$ on the figure) must have completely sent one message before it can start sending another message. This behavior corresponds to the one-port model described in [5]. As the root processor sends data to processors in turn, a receiving processor actually begins its communication after all previous processors have been served. This leads to a "stair effect" represented on Fig. 1 by the end times of the receive operations (black boxes). The implementation also makes the order of the destination processors follow the processors ranks.

As noted by Yang and Casanova [30], the one-port model is a common assumption but in some cases, e.g., for WAN connections, the master could send data to slaves simultaneously to achieve better throughput, and thus a multi-port model could be used. This solution would however require an appropriate implementation for the scatter operation, and would lead to a more complicated problem. For a multi-port solution, a model of the network behavior (in particular when two communications occur in parallel) is needed. This comes to discover the network charac- 
teristics, which is a difficult problem by itself [25]. Indeed we need to know which communications it would be beneficial to do in parallel, before deciding what communications should take place, and when.

\section{Static load-balancing}

In this section, we present different ways to solve the optimal data distribution problem. After briefly presenting our framework, we give two dynamic programming algorithms, the second one being more efficient than the first one, but under some additional hypotheses on the cost functions. We finish by presenting a guaranteed heuristic using linear programming that can be used to quickly find a very good approximation when the cost functions are affine.

As the overall execution time after load-balancing is rather small, we make the assumption that the grid characteristics do not change during the computation and we only consider static load-balancing. Note also that the computed distribution is not necessarily based on static parameters estimated for the whole execution: a monitor daemon process (like [29]) running aside the application could be queried just before a scatter operation to retrieve the instantaneous grid characteristics.

\subsection{Framework}

In this paragraph, we introduce some notations, as well as the cost model used to further derive the optimal data distribution.

We consider a set of $p$ processors: $P_{1}, \ldots, P_{p}$. Processor $P_{i}$ is characterized by (1) the time $T_{\text {comp }}(i, x)$ it takes to compute $x$ data items; (2) the time $T_{\text {comm }}(i, x)$ it takes to receive $x$ data items from the root processor. We want to process $n$ data items. Thus, we look for a distribution $n_{1}, \ldots, n_{p}$ of these data over the $p$ processors that minimizes the overall computation time. All along the paper the root processor will be the last processor, $P_{p}$ (this simplifies expressions as $P_{p}$ can only start to process its share of the data items after it has sent the other data items to the other processors). As the root processor sends data to processors in turn, processor $P_{i}$ begins its communication after processors $P_{1}, \ldots, P_{i-1}$ have been served, which takes a time $\sum_{j=1}^{i-1} T_{\text {comm }}\left(j, n_{j}\right)$. Then the root takes a time $T_{\text {comm }}\left(i, n_{i}\right)$ to send to $P_{i}$ its data. Finally $P_{i}$ takes a time $T_{\text {comp }}\left(i, n_{i}\right)$ to process its share of the data. Thus, $P_{i}$ ends its processing at time:

$$
T_{i}=\sum_{j=1}^{i} T_{\text {comm }}\left(j, n_{j}\right)+T_{\text {comp }}\left(i, n_{i}\right) .
$$

The time, $T$, taken by our system to compute the set of $n$ data items is therefore:

$$
T=\max _{1 \leqslant i \leqslant p} T_{i}=\max _{1 \leqslant i \leqslant p}\left(\sum_{j=1}^{i} T_{\text {comm }}\left(j, n_{j}\right)+T_{\text {comp }}\left(i, n_{i}\right)\right),
$$

and we are looking for the distribution $n_{1}, \ldots, n_{p}$ minimizing this duration. 


\subsection{An exact solution by dynamic programming}

In this section we present two dynamic programming algorithms to compute the optimal data distribution. The first one only assumes that the cost functions are non-negative. The second one presents some optimizations that makes it perform far quicker, but under the additional hypothesis that the cost functions are increasing.

\subsubsection{Basic algorithm}

We now study Eq. (2). The overall execution time is the maximum of the execution time of $P_{1}$, and of the other processors:

$$
T=\max \left(T_{\text {comm }}\left(1, n_{1}\right)+T_{\text {comp }}\left(1, n_{1}\right), \max _{2 \leqslant i \leqslant p}\left(\sum_{j=1}^{i} T_{\text {comm }}\left(j, n_{j}\right)+T_{\text {comp }}\left(i, n_{i}\right)\right)\right) .
$$

Then, one can remark that all the terms in this equation contain the time needed for the root processor to send $P_{1}$ its data. Therefore, Eq. (2) can be written:

$$
T=T_{\text {comm }}\left(1, n_{1}\right)+\max \left(T_{\text {comp }}\left(1, n_{1}\right), \max _{2 \leqslant i \leqslant p}\left(\sum_{j=2}^{i} T_{\text {comm }}\left(j, n_{j}\right)+T_{\text {comp }}\left(i, n_{i}\right)\right)\right) .
$$

So, we notice that the time to process $n$ data on processors 1 to $p$ is equal to the time taken by the root to send $n_{1}$ data to $P_{1}$ plus the maximum of (1) the time taken by $P_{1}$ to process its $n_{1}$ data; (2) the time for processors 2 to $p$ to process $n-n_{1}$ data. This leads to the dynamic programming Algorithm 1 presented on page 7 (the distribution is expressed as a list, hence the use of the list constructor "cons"). In Algorithm $1, \operatorname{cost}[d, i]$ denotes the cost of the processing of $d$ data items over the processors $P_{i}$ through $P_{p}$. solution $[d, i]$ is a list describing a distribution of $d$ data items over the processors $P_{i}$ through $P_{p}$ which achieves the minimal execution time cost $[d, i]$.

Algorithm 1 has a complexity of $\mathrm{O}\left(p \cdot n^{2}\right)$, which may be prohibitive. But Algorithm 1 only assumes that the functions $T_{\text {comm }}(i, x)$ and $T_{\text {comp }}(i, x)$ are non-negative and null whenever $x=0$.

Algorithm 1. Compute an optimal distribution of $n$ data over $p$ processors.

function compute-distribution $(n, p)$

1: $\operatorname{solution}[0, p] \leftarrow \operatorname{cons}(0, N I L)$

2: $\operatorname{cost}[0, p] \leftarrow 0$

3: for $d \leftarrow 1$ to $n$ do

4: $\quad$ solution $[d, p] \leftarrow \operatorname{cons}(d, N I L)$

5: $\operatorname{cost}[d, p] \leftarrow T_{\text {comm }}(p, d)+T_{\text {comp }}(p, d)$

6: end for

7: for $i \leftarrow p-1$ down to 1 do

8: $\quad$ solution $[0, i] \leftarrow \operatorname{cons}(0$, solution $[0, i+1])$ 


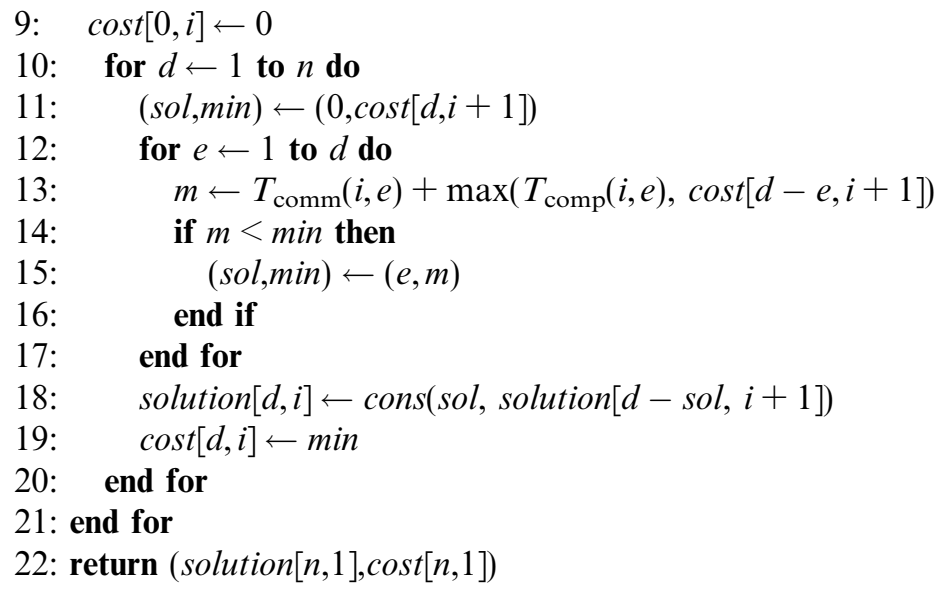

\subsubsection{Optimized algorithm}

If we now make the assumption that $T_{\text {comm }}(i, x)$ and $T_{\text {comp }}(i, x)$ are increasing with $x$, we can make some optimizations on the algorithm. These optimizations consist in reducing the bounds of the inner loop (e-loop, lines 12-17 of Algorithm 1). Algorithm 2, on the next page, presents these optimizations.

Let us explain what changed between the two algorithms. For the following, remember the hypothesis that $T_{\text {comm }}(i, x)$ and $T_{\text {comp }}(i, x)$ are increasing with $x$. As $T_{\text {comm }}(i, x)$ and $T_{\text {comp }}(i, x)$ are non-negative, $\operatorname{cost}[x, i]$ is obviously increasing too, and thus $\operatorname{cost}[d-x, i]$ is decreasing with $x$. The purpose of the $e$-loop is to find $s o l$ in $[0, d]$ such that $T_{\text {comm }}(i, s o l)+\max \left(T_{\text {comp }}(i, s o l), \operatorname{cost}[d-s o l, i+1]\right)$ is minimal. We try (1) to reduce the upper bound of this loop, and (2) to increase the lower bound.

Let $e_{\max }$ be the smallest integer such that $T_{\text {comp }}\left(i, e_{\max }\right) \geqslant \operatorname{cost}\left[d-e_{\max }, i+1\right]$. For all $e \geqslant e_{\max }$, we have $T_{\text {comp }}(i, e) \geqslant T_{\text {comp }}\left(i, e_{\max }\right) \geqslant \operatorname{cost}\left[d-e_{\max }, i+1\right] \geqslant \operatorname{cost}[d-e$, $i+1]$, so $\min _{e} \geqslant e_{\max }\left(T_{\text {comm }}(i, e)+\max \left(T_{\text {comp }}(i, e), \quad \operatorname{cost}[d-e, i+1]\right)\right)$ equals to $\min _{e} \geqslant e_{\max }\left(T_{\text {comm }}(i, e)+T_{\text {comp }}(i, e)\right)$. As $T_{\text {comm }}(i, e)$ and $T_{\text {comp }}(i, e)$ are both increasing with $e, \min _{e} \geqslant e_{\max }\left(T_{\text {comm }}(i, e)+T_{\text {comp }}(i, e)\right)$ equals to $T_{\text {comm }}\left(i, e_{\max }\right)+T_{\text {comp }}\left(i, e_{\text {max }}\right)$. By using a binary search to find $e_{\max }$ (lines 16-26 of Algorithm 2), and by taking care of the cases when $e_{\max }$ falls before 0 (line 12) or after $d$ (line 14), we can reduce the upper bound of the $e$-loop. To take advantage of this information, the direction of the loop must also be inverted. Besides that, we know that inside the loop, cost $[d-e, i+1]$ is always greater than $T_{\text {comp }}(i, e)$, so the max in the computation of $m$ can be avoided (line 29).

We cannot proceed the same way to increase the lower bound of the $e$-loop. We can however remark that, as the loop has been inverted, $e$ is decreasing, so $\operatorname{cost}[d-e$, $i+1]$ is increasing. If $\operatorname{cost}[d-e, i+1]$ becomes greater than or equal to $\mathrm{min}$, then for all $e^{\prime}<e$, we have $\operatorname{cost}\left[d-e^{\prime}, i+1\right] \geqslant \operatorname{cost}[d-e, i+1] \geqslant \min$, and as $T_{\text {comm }}(i, x)$ is non-negative, $T_{\text {comm }}\left(i, e^{\prime}\right)+\operatorname{cost}\left[d-e^{\prime}, i+1\right] \geqslant \min$. The iteration can thus be stopped, hence the break (line 33). 
In the worst case, the complexity of Algorithm 2 is the same than for Algorithm 1, i.e., $\mathrm{O}\left(p \cdot n^{2}\right)$. In the best case, it is $\mathrm{O}(p \cdot n)$. We implemented both algorithms, and in practice Algorithm 2 is far more efficient.

In spite of these optimizations, running the implementation of Algorithm 2 is still time-consuming. Another way to decrease the execution time of these algorithms would be by increasing the granularity, i.e., by grouping data items into blocks and to consider the repartition of these blocks among the processors. This would of course lead to a non-optimal solution. However, since there are very few constraints on the cost functions, it is impossible in the general case to bound the error according to the block size. That is why we now present a more efficient heuristic valid for simple cases.

Algorithm 2. Compute an optimal distribution of $n$ data over $p$ processors (optimized version).

function compute-distribution $(n, p)$

1: $\operatorname{solution}[0, p] \leftarrow \operatorname{cons}(0, N I L)$

2: $\operatorname{cost}[0, p] \leftarrow 0$

3: for $d \leftarrow 1$ to $n$ do

4: $\quad$ solution $[d, p] \leftarrow \operatorname{cons}(d, N I L)$

5: $\quad \operatorname{cost}[d, p] \leftarrow T_{\text {comm }}(p, d)+T_{\text {comp }}(p, d)$

6: end for

7: for $i \leftarrow p-1$ down to 1 do

8: $\quad$ solution $[0, i] \leftarrow \operatorname{cons}(0$, solution $[0, i+1])$

9: $\quad \operatorname{cost}[0, i] \leftarrow 0$

10: $\quad$ for $d \leftarrow 1$ to $n$ do

11: $\quad$ if $T_{\text {comp }}(i, 0) \geqslant \operatorname{cost}[d, i+1]$ then

12: $\quad($ sol, $\min ) \leftarrow\left(0, T_{\text {comm }}(i, 0)+T_{\text {comp }}(i, 0)\right)$

13: $\quad$ else if $T_{\text {comp }}(i, d)<\operatorname{cost}[0, i+1]$ then

14: $\quad(\operatorname{sol}, \min ) \leftarrow\left(d, T_{\text {comm }}(i, d)+\operatorname{cost}[0, i+1]\right)$

15: else

16: $\quad\left(e_{\min }, e_{\max }\right) \leftarrow(0, d)$

17: $\quad e \leftarrow\lfloor d / 2\rfloor$

18: $\quad$ while $e \neq e_{\text {min }}$ do

19: $\quad$ if $T_{\text {comp }}(i, e)<\operatorname{cost}[d-e, i+1]$ then

20: $\quad e_{\min } \leftarrow e$

21: $\quad$ else

22:

23:

24:

25:

$e_{\max } \leftarrow e$

end if

$$
e \leftarrow\left\lfloor\left(e_{\min }+e_{\max }\right) / 2\right\rfloor
$$

end while

26:

27:

$($ sol, $\min ) \leftarrow\left(e_{\max }, T_{\text {comm }}\left(i, e_{\max }\right)+T_{\text {comp }}\left(i, e_{\max }\right)\right)$

\section{end if}

28: $\quad$ for $e \leftarrow s o l-1$ down to 0 do

29: $\quad m \leftarrow T_{\text {comm }}(i, e)+\operatorname{cost}[d-e, i+1]$ 
30: $\quad$ if $m<\min$ then

31: $\quad(\mathrm{sol}, \mathrm{min}) \leftarrow(e, m)$

32: $\quad$ else if $\operatorname{cost}[d-e, i+1] \geqslant \min$ then

33: break

34: $\quad$ end if

35: $\quad$ end for

36: $\quad$ solution $[d, i] \leftarrow \operatorname{cons}(\operatorname{sol}$, solution $[d-\operatorname{sol}, i+1])$

37: $\quad \operatorname{cost}[d, i] \leftarrow \min$

38: end for

39: end for

40: return $(\operatorname{solution}[n, 1], \operatorname{cost}[n, 1])$

\subsection{A guaranteed heuristic using linear programming}

In this section, we consider the realistic but less general case when all communication and computation times are affine functions. This new assumption enables us to code our problem as a linear program. Furthermore, from the linear programming formulation we derive an efficient and guaranteed heuristic.

Thus, we make the hypothesis that all the functions $T_{\text {comm }}(i, n)$ and $T_{\text {comp }}(i, n)$ are affine in $n$, increasing, and non-negative (for $n \geqslant 0$ ). Eq. (2) can then be coded into the following linear program:

$$
\left\{\begin{array}{l}
\text { Minimize } T \text { such that } \\
\forall i \in[1, p], \quad n_{i} \geqslant 0 \\
\sum_{i=1}^{p} n_{i}=n, \\
\forall i \in[1, p], \quad T \geqslant \sum_{j=1}^{i} T_{\text {comm }}\left(j, n_{j}\right)+T_{\text {comp }}\left(i, n_{i}\right) .
\end{array}\right.
$$

We must solve this linear program in integer because we need an integer solution. The integer resolution is however very time-consuming.

Fortunately, a nice workaround exists which provides a close approximation: we can solve the system in rational to obtain an optimal rational solution $n_{1}, \ldots, n_{p}$ that we round up to obtain an integer solution $n_{1}^{\prime}, \ldots, n_{p}^{\prime}$ with $\sum_{i} n_{i}^{\prime}=n$. Let $T^{\prime}$ be the execution time of this solution, $T$ be the time of the rational solution, and $T_{\text {opt }}$ the time of the optimal integer solution. If $\left|n_{i}-n_{i}^{\prime}\right| \leqslant 1$ for any $i$, which is easily enforced by the rounding scheme described below, then:

$$
T_{\mathrm{opt}} \leqslant T^{\prime} \leqslant T_{\mathrm{opt}}+\sum_{j=1}^{p} T_{\text {comm }}(j, 1)+\max _{1 \leqslant i \leqslant p} T_{\text {comp }}(i, 1) .
$$

Indeed,

$$
T^{\prime}=\max _{1 \leqslant i \leqslant p}\left(\sum_{j=1}^{i} T_{\text {comm }}\left(j, n_{j}^{\prime}\right)+T_{\text {comp }}\left(i, n_{i}^{\prime}\right)\right) .
$$


By hypothesis, $T_{\text {comm }}(j, x)$ and $T_{\text {comp }}(j, x)$ are non-negative, increasing, and affine functions. Therefore,

$$
\begin{aligned}
T_{\text {comm }}\left(j, n_{j}^{\prime}\right) & =T_{\text {comm }}\left(j, n_{j}+\left(n_{j}^{\prime}-n_{j}\right)\right) \leqslant T_{\text {comm }}\left(j, n_{j}+\left|n_{j}^{\prime}-n_{j}\right|\right) \\
& \leqslant T_{\text {comm }}\left(j, n_{j}\right)+T_{\text {comm }}\left(j,\left|n_{j}^{\prime}-n_{j}\right|\right) \leqslant T_{\text {comm }}\left(j, n_{j}\right)+T_{\text {comm }}(j, 1)
\end{aligned}
$$

and we have an equivalent upper bound for $T_{\text {comp }}\left(j, n_{j}^{\prime}\right)$. Using these upper bounds to over-approximate the expression of $T^{\prime}$ given by Eq. (5) we obtain:

$$
T^{\prime} \leqslant \max _{1 \leqslant i \leqslant p}\left(\sum_{j=1}^{i}\left(T_{\text {comm }}\left(j, n_{j}\right)+T_{\text {comm }}(j, 1)\right)+T_{\text {comp }}\left(i, n_{i}\right)+T_{\text {comp }}(i, 1)\right),
$$

which implies Eq. (4) knowing that $T_{\text {opt }} \leqslant T^{\prime}, T \leqslant T_{\text {opt }}$, and finally that $T=\max _{1 \leqslant i \leqslant p}\left(\sum_{j=1}^{i} T_{\text {comm }}\left(j, n_{j}\right)+T_{\text {comp }}\left(i, n_{i}\right)\right)$.

\subsubsection{Rounding scheme}

Our rounding scheme is trivial: first we round, to the nearest integer, the non-integer $n_{i}$ which is nearest to an integer. Doing so we obtain $n_{i}^{\prime}$ and we make an approximation error of $e=n_{i}^{\prime}-n_{i}$ (with $|e|<1$ ). If $e$ is negative (resp. positive), $n_{i}$ was underestimated (resp. overestimated) by the approximation. Then we round to its ceiling (resp. floor), one of the remaining $n_{j}$ s which is the nearest to its ceiling $\left\lceil n_{j}\right\rceil$ (resp. floor $\left\lfloor n_{j}\right\rfloor$ ), we obtain a new approximation error of $e=e+n_{j}^{\prime}-n_{j}$ (with $|e|<1$ ), and so on until there only remains to approximate only one of the $n_{i}$ s, say $n_{k}$. Then we let $n_{k}^{\prime}=n_{k}+e$. The distribution $n_{1}^{\prime}, \ldots, n_{p}^{\prime}$ is thus integer, $\sum_{1 \leqslant i \leqslant p} n_{i}^{\prime}=d$, and each $n_{i}^{\prime}$ differs from $n_{i}$ by less than one.

\subsection{Choice of the root processor}

We make the assumption that, originally, the $n$ data items that must be processed are stored on a single computer, denoted $\mathscr{C}$. A processor of $\mathscr{C}$ may or may not be used as the root processor. If the root processor is not on $\mathscr{C}$, then the whole execution time is equal to the time needed to transfer the data from $\mathscr{C}$ to the root processor, plus the execution time as computed by one of the previous algorithms and heuristic. The best root processor is then the processor minimizing this whole execution time, when picked as root. This is just the result of a minimization over the $p$ candidates.

\section{A case study: solving in rational with linear communication and computation times}

In this section we study a simple and theoretical case. This case study will enable us to define a policy on the order in which the processors must receive their data.

We make the hypothesis that all the functions $T_{\text {comm }}(i, n)$ and $T_{\text {comp }}(i, n)$ are linear in $n$. In other words, we assume that there are constants $\lambda_{i}$ and $\mu_{i}$ such that $T_{\text {comm }}(i, n)=\lambda_{i} \cdot n$ and $T_{\text {comp }}(i, n)=\mu_{i} \cdot n$. (Note that $\lambda_{p}=0$ with our hypotheses.) Also, we only look for a rational solution and not an integer one as we should. In other words, we are going to solve our problem in the divisible load framework [10]. 
We show in Section 4.3 that, in this simple case, the processor ordering leading to the shortest execution time is quite simple. Before that we prove in Section 4.2 that there always is an optimal (rational) solution in which all the working processors have the same ending time. We also show the condition for a processor to receive a share of the whole work. As this condition comes from the expression of the execution duration when all processors have to process a share of the whole work and finishes at the same date, we begin by studying this case in Section 4.1. Finally, in Section 4.4, we derive from our case study a guaranteed heuristic for the general case.

\subsection{Execution duration}

Theorem 1 (Execution duration). If we are looking for a rational solution, if each processor $P_{i}$ receives a (non-empty) share $n_{i}$ of the whole set of $n$ data items and if all processors end their computation at a same date $t$, then the execution duration is

$$
t=\frac{n}{\sum_{i=1}^{p} \frac{1}{\lambda_{i}+\mu_{i}} \cdot \prod_{j=1}^{i-1} \frac{\mu_{j}}{\lambda_{j}+\mu_{j}}}
$$

and processor $P_{i}$ receives

$$
n_{i}=\frac{1}{\lambda_{i}+\mu_{i}} \cdot\left(\prod_{j=1}^{i-1} \frac{\mu_{j}}{\lambda_{j}+\mu_{j}}\right) \cdot t
$$

data to process.

Proof. We want to express the execution duration, $t$, and the number of data processor $P_{i}$ must process, $n_{i}$, as functions of $n$. Eq. (2) states that processor $P_{i}$ ends its processing at time: $T_{i}=\sum_{j=1}^{i} T_{\text {comm }}\left(j, n_{j}\right)+T_{\text {comp }}\left(i, n_{i}\right)$. So, with our current hypotheses: $T_{i}=\sum_{j=1}^{i} \lambda_{j} \cdot n_{j}+\mu_{i} \cdot n_{i}$. Thus, $n_{1}=t /\left(\lambda_{1}+\mu_{1}\right)$ and, for $i \in[2, p]$,

$$
T_{i}=T_{i-1}-\mu_{i-1} \cdot n_{i-1}+\left(\lambda_{i}+\mu_{i}\right) \cdot n_{i} .
$$

As, by hypothesis, all processors end their processing at the same time, then $T_{i}=T_{i-1}=t, n_{i}=\mu_{i-1} /\left(\lambda_{i}+\mu_{i}\right) \cdot n_{i-1}$, and we find Eq. (8).

To express the execution duration $t$ as a function of $n$ we just sum Eq. (8) for all values of $i$ in $[1, p]$ :

$$
n=\sum_{i=1}^{p} n_{i}=\sum_{i=1}^{p} \frac{1}{\lambda_{i}+\mu_{i}} \cdot\left(\prod_{j=1}^{i-1} \frac{\mu_{j}}{\lambda_{j}+\mu_{j}}\right) \cdot t,
$$

which is equivalent to Eq. (7).

In the rest of this paper we note:

$$
D\left(P_{1}, \ldots, P_{p}\right)=\frac{1}{\sum_{i=1}^{p} \frac{1}{\lambda_{i}+\mu_{i}} \cdot \prod_{j=1}^{i-1} \frac{\mu_{j}}{\lambda_{j}+\mu_{j}}}
$$

and so we have $t=n \cdot D\left(P_{1}, \ldots, P_{p}\right)$ under the hypotheses of Theorem 1 . 


\subsection{Simultaneous endings}

In this paragraph we exhibit a condition on the costs functions $T_{\text {comm }}(i, n)$ and $T_{\text {comp }}(i, n)$ which is necessary and sufficient to have an optimal rational solution where each processor receives a non-empty share of data, and all processors end at the same date. This tells us when Theorem 1 can be used to find a rational solution to our system.

Theorem 2 (Simultaneous endings). Given $p$ processors, $P_{1}, \ldots, P_{i}, \ldots, P_{p}$, whose communication and computation duration functions $T_{\mathrm{comm}}(i, n)$ and $T_{\mathrm{comp}}(i, n)$ are linear in $n$, there exists an optimal rational solution where each processor receives a non-empty share of the whole set of data, and all processors end their computation at the same date, if and only if

$$
\forall i \in[1, p-1], \quad \lambda_{i} \leqslant D\left(P_{i+1}, \ldots, P_{p}\right)
$$

Proof. The proof is made by induction on the number of processors. If there is only one processor, then the theorem is trivially true. We shall next prove that if the theorem is true for $p$ processors, then it is also true for $p+1$ processors.

Suppose we have $p+1$ processors $P_{1}, \ldots, P_{p+1}$. An optimal solution for $P_{1}, \ldots$, $P_{p+1}$ to compute $n$ data items is obtained by giving $\alpha \cdot n$ items to $P_{1}$ and $(1-\alpha) \cdot n$ items to $P_{2}, \ldots, P_{p+1}$ with $\alpha$ in $[0,1]$. The end date for the processor $P_{1}$ is then $t_{1}(\alpha)=\left(\lambda_{1}+\mu_{1}\right) \cdot n \cdot \alpha$.

As the theorem is supposed to be true for $p$ processors, we know that there exists an optimal rational solution where processors $P_{2}$ to $P_{p+1}$ all work and finish their work simultaneously, if and only if, $\forall i \in[2, p], \lambda_{i} \leqslant D\left(P_{i+1}, \ldots, P_{p+1}\right)$. In this case, by Theorem 1 , the time taken by $P_{2}, \ldots, P_{p+1}$ to compute $(1-\alpha) \cdot n$ data is $(1-\alpha) \cdot n \cdot D\left(P_{2}, \ldots, P_{p+1}\right)$. So, the processors $P_{2}, \ldots, P_{p+1}$ all end at the same date $t_{2}(\alpha)=\lambda_{1} \cdot n \cdot \alpha+k \cdot n \cdot(1-\alpha)=k \cdot n+\left(\lambda_{1}-k\right) \cdot n \cdot \alpha$ with $k=D\left(P_{2}, \ldots\right.$, $\left.P_{p+1}\right)$.

If $\lambda_{1} \leqslant k$, then $t_{1}(\alpha)$ is strictly increasing, and $t_{2}(\alpha)$ is decreasing. Moreover, we have $t_{1}(0)<t_{2}(0)$ and $t_{1}(1)>t_{2}(1)$, thus the whole end date $\max \left(t_{1}(\alpha), t_{2}(\alpha)\right)$ is minimized for an unique $\alpha$ in ]0,1[, when $t_{1}(\alpha)=t_{2}(\alpha)$. In this case, each processor has some data to compute and they all end at the same date.

On the contrary, if $\lambda_{1}>k$, then $t_{1}(\alpha)$ and $t_{2}(\alpha)$ are both strictly increasing, thus the whole end date $\max \left(t_{1}(\alpha), t_{2}(\alpha)\right)$ is minimized for $\alpha=0$. In this case, processor $P_{1}$ has nothing to compute and its end date is 0 , while processors $P_{2}$ to $P_{p+1}$ all end at a same date $k \cdot n$.

Thus, there exists an optimal rational solution where each of the $p+1$ processors $P_{1}, \ldots, P_{p+1}$ receives a non-empty share of the wholeset of data, and all processors end their computation at the same date, if and only if, $\forall i \in[1, p]$, $\lambda_{i} \leqslant D\left(P_{i+1}, \ldots, P_{p+1}\right)$. 
The proof of Theorem 2 shows that any processor $P_{i}$ satisfying the condition $\lambda_{i}>D\left(P_{i+1}, \ldots, P_{p}\right)$ is not interesting for our problem: using it will only increase the whole processing time. Therefore, we just forget those processors and Theorem 2 states that there is an optimal rational solution where the remaining processors are all working and have the same end date.

\subsection{Processor ordering policy}

As we have stated in Section 2.3, the root processor sends data to processors in turn and a receiving processor actually begins its communication after all previous processors have received their shares of data. Moreover, in the MPICH implementation of MPI, the order of the destination processors in scatter operations follows the processor ranks defined by the program(mer). Therefore, setting the processor ranks influence the order in which the processors start to receive and process their share of the whole work. Eq. (7) shows that in our case the overall computation time is not symmetric in the processors but depends on their ordering. Therefore we must carefully defines this ordering in order to speed-up the whole computation. It appears that in our current case, the best ordering is quite simple:

Theorem 3 (Processor ordering policy). When all functions $T_{\text {comm }}(i, n)$ and $T_{\text {comp }}(i, n)$ are linear in $n$, and when we are only looking for a rational solution, then the smallest execution time is achieved when the processors (the root processor excepted) are ordered in decreasing order of their bandwidth (from $P_{1}$, the processor connected to the root processor with the highest bandwidth, to $P_{p-1}$, the processor connected to the root processor with the smallest bandwidth), the last processor being the root processor, and not taking into account the processing powers of the processors.

Proof. We consider any ordering $P_{1}, \ldots, P_{p}$, of the processors, except that $P_{p}$ is the root processor (as we have explained in Section 3.1). We consider any transposition $\pi$ which permutes two neighbors, but let the root processor untouched. In other words, we consider any order $P_{\pi(1)}, \ldots, P_{\pi(p)}$ of the processors such that there exists $k \in[1, p-2], \pi(k)=k+1, \pi(k+1)=k$, and $\forall j \in[1, p] \backslash\{k, k+1\}, \pi(j)=j$ (note that $\pi(p)=p)$.

Without any loss of generality we can assume that in both orderings all processors whose ranks are between $k+2$ and $p$ receive a non-empty share of the whole data set. Indeed, for any $i \in[k+2, p], P_{i}=P_{\pi(i)}$. Hence the condition for $P_{i}$ to receive a non-empty share of the whole data set in the original ordering is the same than the condition for $P_{\pi(i)}$ to receive a non-empty share of the whole data set in the new ordering, according to Theorem 2. Therefore, we do not need to bother considering the processors of rank greater than or equal to $k+2$ which receive an empty share of the data in the original ordering.

We denote by $\Delta(i, p)$ the time needed by the set of processors $P_{i}, P_{i+1}, \ldots, P_{p}$ to optimally process a data of unit size. Symmetrically, we denote by $\Delta_{\pi}(i, p)$ the time needed by the set of processors $P_{\pi(i)}, P_{\pi(i+1)}, \ldots, P_{\pi(p)}$ to optimally process a data of 
unit size. Because of the previous remark, we know that for any value of $i \in[k+2, p], \Delta(i, p)=\Delta_{\pi}(i, p)=D\left(P_{i}, \ldots, P_{p}\right)$.

We ultimately want to show that an optimal solution, when the processors are ordered in decreasing order of their bandwidth, achieves the shortest execution time possible. Proving that $\lambda_{k+1}<\lambda_{k}$ implies that $\Delta(1, p) \geqslant \Delta_{\pi}(1, p)$ will lead to this result. We start by showing that $\Delta(k, p) \geqslant \Delta_{\pi}(k, p)$. We have two cases to study:

(1) In any optimal solution for the ordering $P_{k}, P_{k+1}, P_{k+2}, \ldots, P_{p}$ at most one of the two processors $P_{k}$ and $P_{k+1}$ receives a non-empty share of data.

Any such optimal solution is also feasible under the ordering $P_{k+1}, P_{k}, P_{k+2}, \ldots, P_{p}$. Therefore, $\Delta_{\pi}(k, p) \leqslant \Delta(k, p)$.

(2) There is an optimal solution for the ordering $P_{k}, P_{k+1}, P_{k+2}, \ldots, P_{p}$ in which both $P_{k}$ and $P_{k+1}$ receive a non-empty share of data.

Then, $\Delta(k, p)=D\left(P_{k}, \ldots, P_{p}\right)$. For $\Delta_{\pi}(k, p)$, things are a bit more complicated. If, for any $i$ in $[k, p-1], \lambda_{\pi(i)} \leqslant D\left(P_{\pi(i+1)}, \ldots, P_{\pi(p)}\right)$, Theorems 2 and 1 apply, and thus:

$$
\Delta_{\pi}(k, p)=\frac{1}{\sum_{i=k}^{p} \frac{1}{\lambda_{\pi(i)}+\mu_{\pi(i)}} \cdot \prod_{j=k}^{i-1} \frac{\mu_{\pi(j)}}{\lambda_{\pi(j)}+\mu_{\pi(j)}}} .
$$

On the opposite, if there exists at least one value $i$ in $[k, p-1]$ such that $\lambda_{\pi(i)}>D\left(P_{\pi(i+1)}, \ldots, P_{\pi(p)}\right)$, then Theorem 2 states that the optimal execution time cannot be achieved on a solution where each processor receives a non-empty share of the whole set of data and all processors end their computation at the same date. Therefore, any solution where each processor receives a non-empty share of the whole set of data and all processors end their computation at the same date leads to an execution time strictly greater than $\Delta_{\pi}(k, p)$ and:

$$
\Delta_{\pi}(k, p)<\frac{1}{\sum_{i=k}^{p} \frac{1}{\lambda_{\pi(i)}+\mu_{\pi(i)}} \cdot \prod_{j=k}^{i-1} \frac{\mu_{\pi(j)}}{\lambda_{\pi(j)}+\mu_{\pi(j)}}} .
$$

Eqs. (9) and (10) are summarized by

$$
\Delta_{\pi}(k, p) \leqslant \frac{1}{\sum_{i=k}^{p} \frac{1}{\lambda_{\pi(i)}+\mu_{\pi(i)}} \cdot \prod_{j=k}^{i-1} \frac{\mu_{\pi(j)}}{\lambda_{\pi(j)}+\mu_{\pi(j)}}}
$$

and proving the following implication:

$$
\lambda_{k+1}<\lambda_{k} \Rightarrow \frac{1}{\sum_{i=k}^{p} \frac{1}{\lambda_{\pi(i)}+\mu_{\pi(i)}} \cdot \prod_{j=k}^{i-1} \frac{\mu_{\pi(j)}}{\lambda_{\pi(j)}+\mu_{\pi(j)}}}<\Delta(k, p)
$$

will prove that indeed $\Delta_{\pi}(k, p)<\Delta(k, p)$. Hence, we study the sign of

$$
\sigma=\frac{1}{\sum_{i=k}^{p} \frac{1}{\lambda_{\pi(i)}+\mu_{\pi(i)}} \cdot \prod_{j=k}^{i-1} \frac{\mu_{\pi(j)}}{\lambda_{\pi(j)}+\mu_{\pi(i)}}}-\frac{1}{\sum_{i=k}^{p} \frac{1}{\lambda_{i}+\mu_{i}} \cdot \prod_{j=k}^{i-1} \frac{\mu_{j}}{\lambda_{j}+\mu_{j}}} .
$$

As, in the above expression, both denominators are obviously (strictly) positive, the sign of $\sigma$ is the sign of: 


$$
\sum_{i=k}^{p} \frac{1}{\lambda_{i}+\mu_{i}} \cdot \prod_{j=k}^{i-1} \frac{\mu_{j}}{\lambda_{j}+\mu_{j}}-\sum_{i=k}^{p} \frac{1}{\lambda_{\pi(i)}+\mu_{\pi(i)}} \cdot \prod_{j=k}^{i-1} \frac{\mu_{\pi(j)}}{\lambda_{\pi(j)}+\mu_{\pi(j)}} .
$$

We want to simplify the second sum in Eq. (13). Thus we remark that for any value of $i \in[k+2, p]$ we have:

$$
\prod_{j=k}^{i-1} \frac{\mu_{\pi(j)}}{\lambda_{\pi(j)}+\mu_{\pi(j)}}=\prod_{j=k}^{i-1} \frac{\mu_{j}}{\lambda_{j}+\mu_{j}} .
$$

In order to take advantage of the simplification proposed by Eq. (14), we decompose the second sum in Eq. (13) in three terms: the terms for $k$, for $k+1$, and then the sum from $k+2$ to $p$ :

$$
\begin{aligned}
\sum_{i=k}^{p} \frac{1}{\lambda_{\pi(i)}+\mu_{\pi(i)}} \cdot \prod_{j=k}^{i-1} \frac{\mu_{\pi(j)}}{\lambda_{\pi(j)}+\mu_{\pi(j)}}= & \frac{1}{\lambda_{k+1}+\mu_{k+1}}+\frac{1}{\lambda_{k}+\mu_{k}} \cdot \frac{\mu_{k+1}}{\lambda_{k+1}+\mu_{k+1}} \\
& +\sum_{i=k+2}^{p} \frac{1}{\lambda_{i}+\mu_{i}} \cdot \prod_{j=k}^{i-1} \frac{\mu_{j}}{\lambda_{j}+\mu_{j}} .
\end{aligned}
$$

Then we report the result of Eq. (15) in Eq. (13) and we suppress the terms common to both sides of the "-" sign. This way, we obtain that $\sigma$ has the same sign than:

$$
\frac{1}{\lambda_{k}+\mu_{k}}+\frac{1}{\lambda_{k+1}+\mu_{k+1}} \cdot \frac{\mu_{k}}{\lambda_{k}+\mu_{k}}-\frac{1}{\lambda_{k+1}+\mu_{k+1}}-\frac{1}{\lambda_{k}+\mu_{k}} \cdot \frac{\mu_{k+1}}{\lambda_{k+1}+\mu_{k+1}},
$$

which is equivalent to:

$$
\frac{\lambda_{k+1}-\lambda_{k}}{\left(\lambda_{k}+\mu_{k}\right) \cdot\left(\lambda_{k+1}+\mu_{k+1}\right)} \text {. }
$$

Therefore, if $\lambda_{k+1}<\lambda_{k}$, then $\sigma<0$, Eq. (12) holds, and thus $\Delta_{\pi}(k, p)<\Delta(k, p)$. Note that if $\lambda_{k+1}=\lambda_{k}, \sigma=0$ and thus $\Delta_{\pi}(k, p) \leqslant \Delta(k, p)$. As we have made no assumptions on the computational powers of $P_{k}$ and $P_{k+1}$, this symmetrically shows that $\Delta_{\pi}(k, p) \geqslant \Delta(k, p)$ and thus that $\Delta_{\pi}(k, p)=\Delta(k, p)$. Therefore, processors which are connected with the same bandwidth can be set in any order.

We have just proved that $\Delta_{\pi}(k, p) \leqslant \Delta(k, p)$. We now prove by induction that for any value of $i \in[1, k], \Delta_{\pi}(i, p) \leqslant \Delta(i, p)$. Once we will have established this result, we will have that $\Delta_{\pi}(1, p) \leqslant \Delta(1, p)$ which is exactly our goal.

Let us suppose that the induction is proved from $k$ down to $i$, and focus on the case $i-1$. We have three cases to consider:

(1) $\lambda_{i-1} \leqslant \Delta_{\pi}(i, p) \leqslant \Delta(i, p)$.

Then, following the proof of Theorem 2, we have:

$$
\Delta_{\pi}(i-1, p)=\frac{1}{\frac{1}{\lambda_{i-1}+\mu_{i-1}}+\frac{\mu_{i-1}}{\left(\lambda_{i-1}+\mu_{i-1}\right) \cdot \Delta_{\pi}(i, p)}}
$$


and an equivalent formula for $\Delta(i-1, p)$. Therefore, $\Delta_{\pi}(i, p) \leqslant \Delta(i, p)$ implies $\Delta_{\pi}(i-1, p) \leqslant \Delta(i-1, p)$.

(2) $\Delta_{\pi}(i, p) \leqslant \lambda_{i-1} \leqslant \Delta(i, p)$.

Then, $\Delta_{\pi}(i-1, p)=\Delta_{\pi}(i, p)$ and $\Delta(i-1, p)=\frac{\left(\lambda_{i-1}+\mu_{i-1}\right) \cdot \Delta(i, p)}{\Delta(i, p)+\mu_{i-1}}$. Then,

$$
\Delta(i-1, p)-\Delta_{\pi}(i-1, p)=\frac{\mu_{i-1} \cdot\left(\Delta(i, p)-\Delta_{\pi}(i, p)\right)+\left(\lambda_{i-1}-\Delta_{\pi}(i, p)\right) \cdot \Delta(i, p)}{\Delta(i, p)+\mu_{i-1}} .
$$

Then, because of the hypothesis on $\lambda_{i-1}$ and because of the induction hypothesis, $\Delta(i-1, p)-\Delta_{\pi}(i-1, p)$ is non-negative.

(3) $\Delta_{\pi}(i, p) \leqslant \Delta(i, p)<\lambda_{i-1}$.

Then the result is obvious as $\Delta_{\pi}(i-1, p)=\Delta_{\pi}(i, p)$ and $\Delta(i, p)=\Delta(i-1, p)$.

Therefore, the inversion of processors $P_{k}$ and $P_{k+1}$ is profitable if the bandwidth from the root processor to processor $P_{k+1}$ is higher than the bandwidth from the root processor to processor $P_{k}$.

\subsection{Consequences for the general case}

So, in the general case, how are we going to order our processors? An exact study is feasible even in the general case, if we know the computation and communication characteristics of each of the processors. We can indeed consider all the possible orderings of our $p$ processors, use Algorithm 1 to compute the theoretical execution times, and chose the best result. This is theoretically possible. In practice, for large values of $p$ such an approach is unrealistic. Furthermore, in the general case an analytical study is of course impossible (we cannot analytically handle any function $T_{\text {comm }}(i, n)$ or $\left.T_{\text {comp }}(i, n)\right)$.

So, we build from the previous result and we order the processors in decreasing order of the bandwidth they are connected to the root processor with, except for the root processor which is ordered last. Even without the previous study, such a policy should not be surprising. Indeed, the time spent to send its share of the data items to processor $P_{i}$ is payed by all the processors from $P_{i}$ to $P_{p}$. So the first processor should be the one it is the less expensive to send the data to, and so on. Of course, in practice, things are a bit more complicated as we are working in integers. However, the main idea is roughly the same as we now show.

We only suppose that all the computation and communication functions are linear. Then we denote by

- $T_{\mathrm{opt}}^{\mathrm{rat}}$ : the best execution time that can be achieved for a rational distribution of the $n$ data items, whatever the ordering for the processors.

- $T_{\mathrm{opt}}^{\mathrm{int}}$ : the best execution time that can be achieved for an integer distribution of the $n$ data items, whatever the ordering for the processors.

Note that $T_{\mathrm{opt}}^{\mathrm{rat}}$ and $T_{\mathrm{opt}}^{\mathrm{int}}$ may be achieved on two different orderings of the processors. We take a rational distribution achieving the execution time $T_{\mathrm{opt}}^{\text {rat }}$. We round it up to 
obtain an integer solution, following the rounding scheme described in Section 3.3. This way we obtain an integer distribution of execution time $T^{\prime}$ with $T^{\prime}$ satisfying the equation:

$$
T^{\prime} \leqslant T_{\mathrm{opt}}^{\mathrm{rat}}+\sum_{j=1}^{p} T_{\text {comm }}(j, 1)+\max _{1 \leqslant i \leqslant p} T_{\text {comp }}(i, 1)
$$

(the proof being the same than for Eq. (4)). However, as it is an integer solution, its execution time is obviously at least equal to $T_{\mathrm{opt}}^{\mathrm{int}}$. Also, an integer solution being a rational solution, $T_{\mathrm{opt}}^{\mathrm{int}}$ is at least equal to $T_{\mathrm{opt}}^{\mathrm{rat}}$. Hence the bounds:

$$
T_{\mathrm{opt}}^{\mathrm{int}} \leqslant T^{\prime} \leqslant T_{\mathrm{opt}}^{\mathrm{int}}+\sum_{j=1}^{p} T_{\text {comm }}(j, 1)+\max _{1 \leqslant i \leqslant p} T_{\text {comp }}(i, 1),
$$

where $T^{\prime}$ is the execution time of the distribution obtained by rounding up, according to the scheme of Section 3.3, the best rational solution when the processors are ordered in decreasing order of the bandwidth they are connected to the root processor with, except for the root processor which is ordered last. Therefore, when all the computation and communication functions are linear our ordering policy is even guaranteed!

\section{Experimental results}

\subsection{Hardware environment}

Our experiment consists in the computation of 817,101 ray paths (the full set of seismic events of year 1999) on 16 processors. All machines run Globus [15] and we use MPICH-G2 [22] as message passing library. Table 1 shows the resources used in the experiment. The input data set is located on the PC named dinadan, at the end of the list, which serves as root processor. Except for the root, the resources are ordered in decreasing order of their bandwidths to the root processor. The computers are located at two geographically distant sites. Processors 1, 2, 3, 16 (standard PCs with Intel PIII and AMD Athlon XP), and 4, 5 (two Mips processors of an SGI Origin 2000) are in the same premises, whereas processors 6-13 are taken from an SGI

Table 1

Processors used as computational nodes in the experiment

\begin{tabular}{llllll}
\hline Machine & CPU \# & Type & $\mu(\mathrm{s} /$ ray $)$ & Rating & $\lambda(\mathrm{s} / \mathrm{ray})$ \\
\hline caseb & 1 & XP1800 & 0.004629 & 2.00 & $1.00 \times 10^{-5}$ \\
pellinore & 2 & PIII/800 & 0.009365 & 0.99 & $1.12 \times 10^{-5}$ \\
sekhmet & 3 & XP1800 & 0.004885 & 1.90 & $1.70 \times 10^{-5}$ \\
seven & 4,5 & R12K/300 & 0.016156 & 0.57 & $2.10 \times 10^{-5}$ \\
leda & $6-13$ & R14K/500 & 0.009677 & 0.95 & $3.53 \times 10^{-5}$ \\
merlin & 14,15 & XP2000 & 0.003976 & 2.33 & $8.15 \times 10^{-5}$ \\
dinadan & 16 & PIII/933 & 0.009288 & 1.00 & $0.00 \times 10^{-5}$ \\
\hline
\end{tabular}


Origin 3800 (Mips processors) named leda, at the other end of France. The processors and the network links performances are values computed from a series of benchmarks we performed on our application. Notice that merlin, with processors 14 and 15 , though geographically close to the root processor, has the smallest bandwidth because it was connected to a $10 \mathrm{Mbit} / \mathrm{s}$ hub during the experiment whereas all others were connected to fast-ethernet switches.

The column $\mu$ indicates the number of seconds needed to compute one ray (the lower, the better). The associated rating is simply a more intuitive indication of the processor speed (the higher, the better): it is the inverse of $\mu$ normalized with respect to a rating of 1 arbitrarily chosen for the Pentium III/933. When several identical processors are present on a same computer $(6-13$ and 14, 15) the average performance is reported.

The network links throughputs between the root processor and the other nodes are reported in column $\lambda$ assuming a linear communication cost. It indicates the time in seconds needed to receive one data element from the root processor. Considering linear communication costs is sufficiently accurate in our case since the network latency is negligible compared to the sending time of the data blocks.

\subsection{Results}

The experimental results of this section evaluate two aspects of the study. The first experiment compares an imbalanced execution (that is the original program without any source code modification) to what we predict to be the best balanced execution. The second experiment evaluates the execution performances with respect to our processor ordering policy (the processors are ordered in descending order of their bandwidths) by comparing this policy to the opposite one (the processors are ordered in ascending order of their bandwidths). Note that whatever the order chosen, the root processor is always set at the end of the list (despite its infinite bandwidth to itself) since the scatter semantics forces this processor to receive its own data share last (see Section 3.1).

\subsubsection{Original application}

Fig. 2 reports performance results obtained with the original program, in which each processor receives an equal amount of data. We had to choose an ordering of the processors, and from the conclusion given in Section 4.4, we ordered processors by descending bandwidth.

Not surprisingly, the processors end times largely differ, exhibiting a huge imbalance, with the earliest processor finishing after $241 \mathrm{~s}$ and the latest after $835 \mathrm{~s}$.

\subsubsection{Load-balanced application}

In the second experiment we evaluate our load-balancing strategy. We made the assumption that the computation and communication cost functions were affine and increasing. This assumption allowed us to use our guaranteed heuristic. Then, we simply replaced the MPI_Scatter call by a MPI_Scatterv parameterized with the distribution computed by the heuristic. With such a large number of rays, 


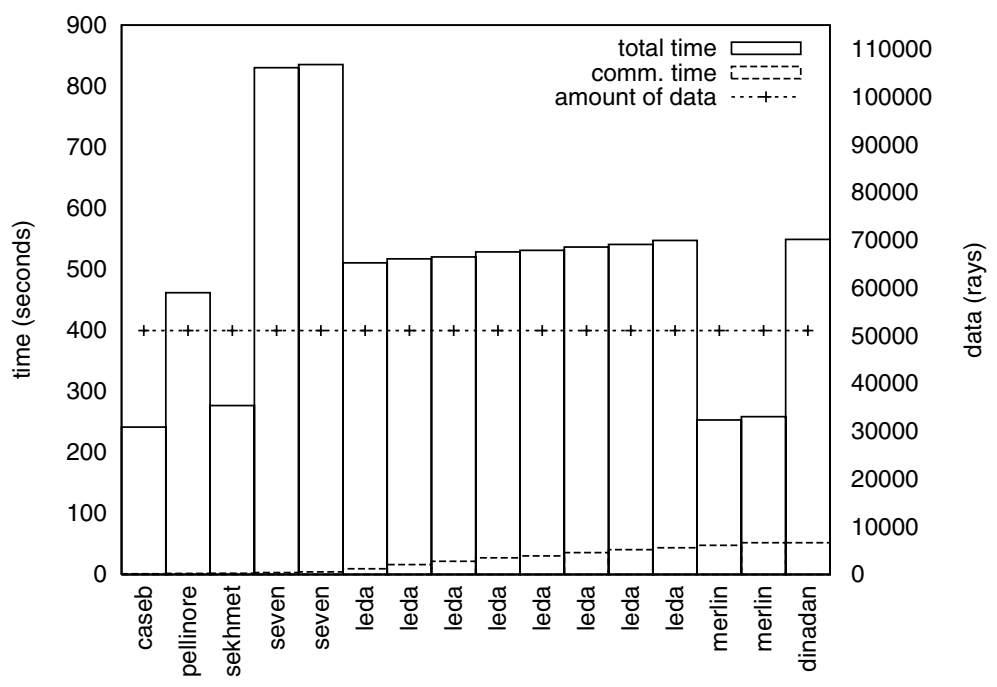

Fig. 2. Original program execution (uniform data distribution).

Algorithm 1 takes more than two days of work (we interrupted it before its completion) and Algorithm 2 takes 6 min to run on a Pentium III/933 whereas the heuristic execution, using pipMP [14,27], is instantaneous and has an error relative to the optimal solution of less than $6 \times 10^{-6}$ !

Results of this experiment are presented in Fig. 3. The execution appears well balanced: the earliest and latest finish times are $388 \mathrm{~s}$ and $412 \mathrm{~s}$ respectively, which

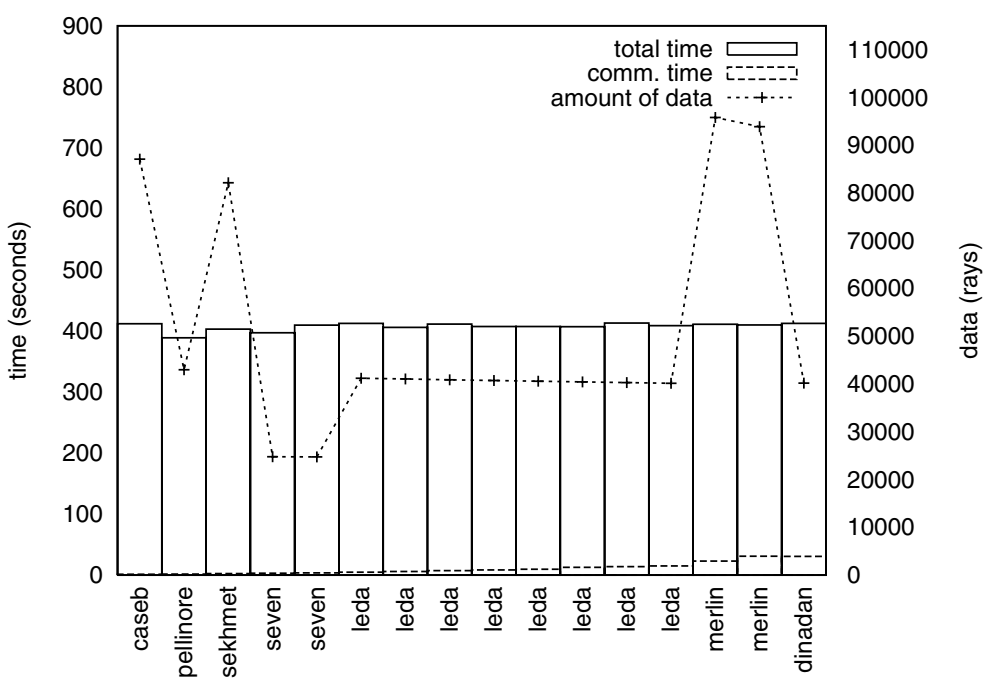

Fig. 3. Load-balanced execution with nodes sorted by descending bandwidth. 
represents a maximum difference in finish times of $6 \%$ of the total duration. By comparison to the performances of the original application, the gain is significant: the total execution duration is approximately half the duration of the first experiment.

\subsubsection{Ordering policy}

We now compare the effects of the ordering policy. Results presented in Fig. 3 were obtained with the descending bandwidth order. The same execution with processors sorted in ascending bandwidth order is presented in Fig. 4.

The load balance in this execution is acceptable with a maximum difference in ending times of about $10 \%$ of the total duration (the earliest and latest processors finish after $420 \mathrm{~s}$ and $469 \mathrm{~s}$ ). As predicted, the total duration is longer (by $57 \mathrm{~s}$ ) than with the processors in the reverse order. Though the load was slightly less balanced than in the previous experiment (because of a peak load on sekhmet during the experiment), most of the difference comes from the idle time spent by processors waiting before the actual communication begins. This clearly appears in Fig. 4: the surface of the bottom area delimited by the dashed line (the "stair effect") is bigger than in Fig. 3.

\subsubsection{Predicted times}

If we now look at the differences between the times predicted by using our model (see Section 3.1) and the completion times achieved in reality, we can see that the accuracy of the predictions depends on the processors characteristics, and more precisely on the possible interferences that can occur during the execution.

For the communication times, the predictions were more accurate for the processors that were geographically close to the root with an error that was less than $0.7 \mathrm{~s}$, while it was up to $5.5 \mathrm{~s}$ for the processors located at the other end of the country. This certainly comes from the fact that network performances are more stable

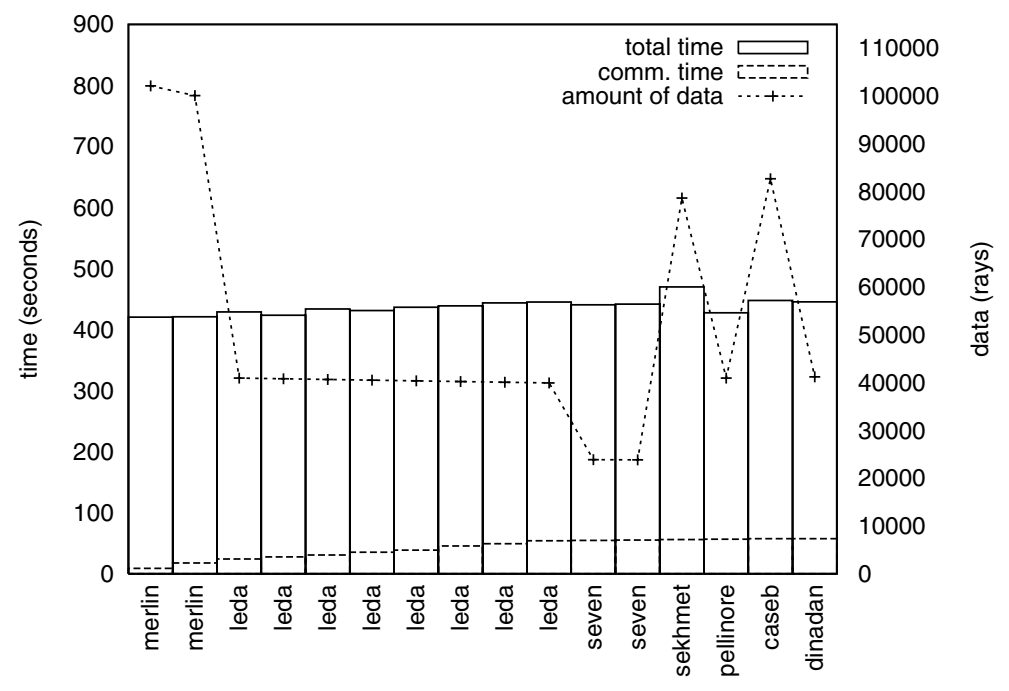

Fig. 4. Load-balanced execution with nodes sorted by ascending bandwidth. 
(and thus more easily predictable) for a local network than for a wide area network. Compared to the small communication times we had, these errors look rather big. We can however see, from Figs. 3 and 4, that, despite the possible mispredictions, it is important to take the communications into account to choose a good processor ordering and to compute a load-balanced data distribution. For the computation times, the relative error was less than $2.3 \%$ on the Origins (leda and seven) while it was sometimes as great as $9.2 \%$ on the PCs. The difference here is due to the fact that the processors on the PCs were possibly shared with other users while a reservation system (LSF in this case) gave us a dedicated use of the processors on the parallel machines. Concerning the whole execution time of the experiments, the prediction was pretty good for the first two experiments: it was underestimated by less than $1.9 \%$. As already seen for the load-balancing, some interferences took place during the third experiment, leading here to an underestimation of about $11.6 \%$. With a static approach like the one presented in this paper, the quality of the prediction clearly depends on the predictability of the network links bandwidths and the processors performances. These experiments show however that a good load-balance along with a close prediction of the end time are achievable in practice.

\section{Related work}

Many research works address the problem of load-balancing in heterogeneous environments, but most of them consider dynamic load-balancing. As a representative of the dynamic approach, the framework developed by [18] applies to iterative SPMD computations, i.e. similar computations are performed iteratively on independent data items. In this work, a library helps the programmer to distribute data among processors and collects processors performances at each iteration. Based on these performances, the programmer may, between two iterations, decide to redistribute some of the data among the processors so as to load-balance the following iterations. Note that the initial distribution computation of data as well as the redistribution decision is left to the programmer who can plug-in its own distribution algorithm. Anyhow, the load evaluation and the data redistribution make the execution suffer from overheads that can be avoided with a static approach. In a previous work [13], we experimented dynamic load-balancing with a master-slave implementation of the geophysical application, and compared it with a static version. The dynamic version has the well-known advantage of adapting itself to sudden changes of the load. The drawback lies in the difficulty to choose the data packet size to send to slaves: small packets incur latency penalties for each packet (may be prohibitive on slow networks) and large packets increase the load-imbalance. After having manually tuned the packet size, the dynamic version showed a load-balance which was acceptable but which was still significantly worse than the load-balance of the static version (the imbalance of the dynamic version was roughly twice the imbalance of the static one, in terms of difference of processor completion times).

The static approach is used in various contexts. It ranges from data partitioning for parallel video processing [2] to finding the optimal number of processors in linear 
algebra algorithms [4]. More generally, many results have been produced by the divisible load theory for various network topologies (see [10] for an overview). The contents of Section 4 fits in the divisible load framework. Our hardware model corresponds to the single-level tree network without front-end processors of [9]: the root processor must wait for all the slave processors to have received their respective shares of the data before it can start to process its own share of the computations. For these networks, when the order on the processors is fixed, Robertazzi $[28,9]$ established a condition for a processor to participate in an optimal solution, and he showed that there was an optimal solution where all participating processors ends their computation at the same date. These results are stated by our Theorems 1 and 2. Similar results [9] exist for single-level tree networks with front-end processors: the root processor can compute while sending data to slave processors. In this framework, Kim et al. pretended they showed that, in an optimal solution, the processors are also ordered by decreasing bandwidth [24]. Their proof relies on the unproved assumption that in an optimal solution all processors participate. The same mistake was also made in the same context by Błażewicz and Drozdowski in [11]. Beaumont, Legrand, and Robert [7,8] proved, for the single-level tree networks with front-end processors, that in any optimal solution the processors are ordered by decreasing bandwidth, they all participate in the computation, and all finish their execution at the same time. Beaumont, Legrand, and Robert also proposed asymptotically optimal multi-round algorithms.

In [1], Almeida et al. present a dynamic programming algorithm similar to ours to solve the master-slave problem on heterogeneous systems. The main difference with our work is that they consider results to be sent back to the master. In their model, the master does not compute anything, and he cannot send and receive data simultaneously. A major drawback with their work is that they simply assume that the processors should be ordered by non-increasing order of their computational power (fastest processors first), while several authors have shown that in many cases, the sort criterion should be the network links bandwidths [24,5,7,17].

With a problem formulation very close to ours, Beaumont et al. give in [6], a polynomial-time solution using a non-trivial greedy algorithm. They however restrict to linear cost functions, while our solution presented in Section 3.2 is valid for any nonnegative cost functions. Another difference is in the produced schedule: we constrain that for each slave all the data are sent before starting the computation, whereas they allow communications between different slaves to be interleaved. The same authors, in association with Banino, have studied in [3] more general tree-structured networks where, rather than searching an optimal solution to the problem, they characterize the best steady-state for various operation models.

A related problem is the distribution of loops for heterogeneous processors so as to balance the work-load. This problem is studied in [12], in particular the case of independent iterations, which is equivalent to a scatter operation. However, computation and communication cost functions are affine. A load-balancing solution is first presented for heterogeneous processors, only when no network contentions occur. Then, the contention is taken into account but for homogeneous processors only. 


\section{Conclusion}

In this paper we partially addressed the problem of adapting to the grid existing parallel applications designed for parallel computers. We studied the static loadbalancing of scatter operations when no assumptions are made on the processor speeds or on the network links bandwidth. We presented two solutions to compute load-balanced distributions: a general and exact algorithm, and a heuristic far more efficient for simple cases with a large number of tasks (affine computation and communication times). We also proposed a policy on the processor ordering: we order them in decreasing order of the network bandwidth they have with the root processor. On our target application, our experiments showed that replacing MPI_Scatter by MPI_Scatterv calls used with clever distributions leads to great performance improvement at low cost.

\section{Acknowledgments}

A part of the computational resources used are taken from the Origin 3800 of the CINES (http://www.cines.fr/). We want to thank them for letting us have access to their machines.

This research is partially supported by the French Ministry of Research through the ACI-GRID program.

\section{References}

[1] F. Almeida, D. González, L.M. Moreno, The master-slave paradigm on heterogeneous systems: a dynamic programming approach for the optimal mapping, in: 12th Euromicro Conference on Parallel, Distributed and Network-Based Processing (PDP 2004), IEEE CS Press, 2004, pp. 266-272.

[2] D.T. Altilar, Y. Paker, Optimal scheduling algorithms for communication constrained parallel processing, in: Euro-Par 2002, Parallel Processing, 8th International Euro-Par Conference, Lecture Notes in Computer Science, vol. 2400, Springer-Verlag, 2002, pp. 197-206.

[3] C. Banino, O. Beaumont, A. Legrand, Y. Robert, Scheduling strategies for master-slave tasking on heterogeneous processor grids, in: Applied Parallel Computing: Advanced Scientific Computing: 6th International Conference (PARA'02), Lecture Notes in Computer Science, vol. 2367, Springer-Verlag, 2002, pp. 423-432.

[4] J.G. Barbosa, J. Tavares, A.J. Padilha, Linear algebra algorithms in heterogeneous cluster of personal computers, in: 9th Heterogeneous Computing Workshop (HCW'2000), IEEE CS Press, 2000, pp. 147-159.

[5] O. Beaumont, L. Carter, J. Ferrante, A. Legrand, Y. Robert, Bandwidth-centric allocation of independent tasks on heterogeneous platforms, in: International Parallel and Distributed Processing Symposium (IPDPS'02), IEEE CS Press, 2002.

[6] O. Beaumont, A. Legrand, Y. Robert, A polynomial-time algorithm for allocating independent tasks on heterogeneous fork-graphs, in: 17th International Symposium on Computer and Information Sciences (ISCIS XVII), CRC Press, 2002, pp. 115-119.

[7] O. Beaumont, A. Legrand, Y. Robert, Optimal algorithms for scheduling divisible workloads on heterogeneous systems, in: 12th Heterogeneous Computing Workshop (HCW'2003), IEEE CS Press, 2003. 
[8] O. Beaumont, A. Legrand, Y. Robert, Scheduling divisible workloads on heterogeneous platforms, Parallel Comput. 29 (9) (2003) 1121-1152.

[9] V. Bharadwaj, D. Ghose, V. Mani, T.G. Robertazzi, Scheduling Divisible Loads in Parallel and Distributed Systems, Wiley-IEEE CS Press, 1996.

[10] V. Bharadwaj, D. Ghose, T.G. Robertazzi, Divisible load theory: a new paradigm for load scheduling in distributed systems, Cluster Comput. 6 (1) (2003) 7-17.

[11] J. Błażewicz, M. Drozdowski, Distributed processing of divisible jobs with communication startup costs, Discrete Appl. Math. 76 (1-3) (1997) 21-41.

[12] M. Cierniak, M.J. Zaki, W. Li, Compile-time scheduling algorithms for heterogeneous network of workstations, Comput. J. 40 (6) (1997) 356-372, Special Issue on Automatic Loop Parallelization.

[13] R. David, S. Genaud, A. Giersch, B. Schwarz, É. Violard, Source code transformations strategies to load-balance grid applications, in: Grid Computing — GRID 2002: Third International Workshop, Lecture Notes in Computer Science, vol. 2536, Springer-Verlag, 2002, pp. 82-87.

[14] P. Feautrier, Parametric integer programming, RAIRO Rech. Opér. 22 (3) (1988) 243-268.

[15] I. Foster, C. Kesselman, Globus: a metacomputing infrastructure toolkit, Int. J. Supercomput. Appl. High Perform. Comput. 11 (2) (1997) 115-128.

[16] I. Foster, C. Kesselman (Eds.), The Grid: Blueprint for a New Computing Infrastructure, Morgan Kaufmann, 1999.

[17] S. Genaud, A. Giersch, F. Vivien, Load-balancing scatter operations for grid computing, in: 12th Heterogeneous Computing Workshop (HCW'2003), IEEE CS Press, 2003.

[18] W.L. George, Dynamic load-balancing for data-parallel MPI programs, in: Message Passing Interface Developer's and User's Conference (MPIDC'99), 1999.

[19] M. Grunberg, S. Genaud, C. Mongenet, Seismic ray-tracing and earth mesh modeling on various parallel architectures, J. Supercomput. 29 (1) (2004) 27-44.

[20] P. Husbands, J.C. Hoe, MPI-StarT: delivering network performance to numerical applications, in: Proceedings of the 1998 ACM/IEEE conference on Supercomputing (SC'98), IEEE CS Press, 1998.

[21] N.T. Karonis, B.R. de Supinski, I. Foster, W. Gropp, E. Lusk, J. Bresnahan, Exploiting hierarchy in parallel computer networks to optimize collective operation performance, in: International Parallel and Distributed Processing Symposium (IPDPS'00), IEEE CS Press, 2000, pp. 377-384.

[22] N.T. Karonis, B. Toonen, I. Foster, MPICH-G2: a grid-enabled implementation of the message passing interface, J. Parallel Distrib. Comput. 63 (5) (2003) 551-563.

[23] T. Kielmann, R.F.H. Hofman, H.E. Bal, A. Plaat, R.A.F. Bhoedjang, MagPIe: MPI's collective communication operations for clustered wide area systems, ACM SIGPLAN Notices 34 (8) (1999) $131-140$

[24] H.J. Kim, G.-I. Jee, J.G. Lee, Optimal load distribution for tree network processors, IEEE Trans. Aerosp. Electron. Syst. 32 (2) (1996) 607-612.

[25] A. Legrand, F. Mazoit, M. Quinson, An Application-Level Network Mapper, Research Report 200309, LIP, ENS Lyon, France, February 2003.

[26] MPI Forum. MPI: A message passing interface standard, version 1.1. Technical report, University of Tennessee, Knoxville, TN, USA, June 1995.

[27] PIP/PipLib. URL < http://www.prism.uvsq.fr/cedb/bastools/piplib.html>.

[28] T.G. Robertazzi, Processor equivalence for a linear daisy chain of load sharing processors, IEEE Trans. Aerosp. Electron. Syst. 29 (4) (1993) 1216-1221.

[29] R. Wolski, N.T. Spring, J. Hayes, The network weather service: a distributed resource performance forecasting service for metacomputing, Future Gener. Comput. Syst. 15 (5-6) (1999) 757-768.

[30] Y. Yang, H. Casanova, A multi-round algorithm for scheduling divisible workload applications: analysis and experimental evaluation, Technical Report CS2002-0721, Department of Computer Science and Engineering, University of California, San Diego, September 2002. 